\title{
INTRUMENTOS FINANCEIROS DERIVATIVOS: COMPARAÇÃO DO NÍVEL DE DISCLO- SURE DAS EMPRESAS COM O CONTEÚDO OFERTADO PELOS CURSOS DE GRADUA- ÇÃO EM CIÊNCIAS CONTÁBEIS ${ }^{1}$
}

\section{DERIVATIVE FINANCIAL INSTRUMENTS: COMPARISON OF THE LEVEL OF DISCLOSURE OF COMPANIES WITH THE CONTENT OFFERED BY THE GRADUATE COURSES IN AC- COUNTING SCIENCES}

\author{
Aloisio Pereira Júnior \\ Mestre em Ciências Contábeis (Universidade Federal de Uberlândia) \\ Instituto Federal do Triângulo Mineiro \\ aloisio@iftm.edu.br \\ Rodrigo Fernandes Malaquias \\ Doutorado em Administração (FGV/EASP) \\ Universidade Federal de Uberlândia \\ rodrigofmalaquias@gmail.com
}

\section{RESUMO}

Objetivo: Analisar a relação entre o nível de aderência do disclosure de empresas listadas na B3 e o conteúdo curricular ofertado pelos cursos de graduação em ciências contábeis sobre instrumentos financeiros derivativos.

Fundamento: No atual ambiente de negócios complexo e dinâmico, as empresas vêm utilizando instrumentos financeiros derivativos para a gestão e mitigação dos riscos financeiros e, segundo a norma contábil, esses instrumentos devem ser evidenciados nas demonstrações contábeis. Conforme pesquisas anteriores demonstram, o ensino das normas de contabilidade pode refletir na qualidade das demonstrações contábeis.

Método: Com base nos pronunciamentos técnicos CPC 39, 40 e 48 do Comitê de Pronunciamentos Contábeis, elaborou-se um instrumento de coleta de dados com 40 itens. Estes itens foram utilizados, então, para analisar i) as notas explicativas do ano de 2017 relativas aos exercícios contábeis de 2016 e 2017 de 44 empresas (carteira teórica do Ibovespa do quarto trimestre de 2017); bem como ii) os projetos pedagógicos e fichas de disciplinas de 250 cursos de graduação em Ciências Contábeis brasileiros.

Resultados: Os resultados mostram que não necessariamente os itens com menor aderência de conteúdo dos cursos são os de menor aderência nas notas explicativas, exceto para os itens relacionados a operações e contabilidade de hedge, cujos índices de aderência foram os menores tanto na oferta de seu conteúdo quanto na divulgação de suas informações pelas empresas.

\footnotetext{
${ }^{1}$ Artigo recebido em: 18/03/2019. Revisado por pares em: 08/09/2019. Reformulado em: 27/11/2019. Recomendado para publicação: 03/12/2019 por Adriana Vasconcelos (Editora Chefe). Publicado em: 01/05/2020. Organização responsável pelo periódico: UFPB
} 
Contribuições: $O$ trabalho evidencia a necessidade de melhorias na divulgação das informações sobre o tratamento contábil dos instrumentos financeiros derivativos designados como hedge por parte das empresas estudadas.

Palavras-chave: Instrumentos Financeiros. Derivativos. Disclosure.

\section{ABSTRACT}

Objective: To analyze the relationship between the degree of adherence of the disclosure of companies listed in B3 and the curricular content offered by undergraduate courses in accounting sciences on derivative financial instruments.

Rationale: In the current complex and dynamic business environment, companies have been using derivative financial instruments to manage and mitigate financial risks and, according to accounting standards, these instruments must be evidenced in the financial statements. As previous research has shown, the teaching of accounting standards may reflect on the quality of financial statements.

Method: Based on technical pronouncements CPC 39, 40 and 48 of the Accounting Pronouncements Committee, a data collection instrument with 40 items was prepared. These items were then used to analyze i) the explanatory notes for 2017 for the 2016 and 2017 accounting periods of 44 companies (theoretical portfolio of the Ibovespa of the fourth quarter of 2017); as well as ii) the pedagogical projects and records of 250 undergraduate courses in Brazilian Accounting Sciences.

Results: The results show that not necessarily the items with less adherence of content of the courses are those of less adherence in the explanatory notes, except for the items related to operations and hedge accounting, whose adherence indexes were the smaller both in the offer of its and disclosure of information by companies.

Contributions: The work shows the need for improvements in the disclosure of information on the accounting treatment of derivative financial instruments designated as hedge by the companies studied.

Keywords: Financial Instruments. Derivatives. Disclosure.

\section{INTRODUÇÃO}

No atual mercado globalizado, dinâmico e instável, no curso de suas atividades, as empresas estão sujeitas a diversos riscos financeiros (Amaral, 2003). Para a mitigação dos riscos a que estão expostas, as empresas vêm utilizando instrumentos financeiros derivativos em sua gestão de riscos financeiros (Malaquias \& Lemes, 2013).

Derivativos são instrumentos derivados de um ativo que podem ser contratados pelas empresas para designação de hedge (proteção) com a finalidade de gerenciamento de riscos ou para especulação (Amaral, 2003). A gestão de risco está mais complexa e desafiadora, tornando-se uma tarefa mais sofisticada (Kota \& Charumathi, 2018).

Assim, com um ambiente de negócios mais complexo e em constante mudança, surge a necessidade de se criar normas de divulgação confiáveis e relevantes para auxiliar os usuários contábeis na tomada de decisão (Kota \& Charumathi, 2018) e na compreensão sobre as particularidades relacionadas ao uso de derivativos nas empresas em que investem (ou que possam vir a investir). Como consequência, aumentando a demanda de transparência na divulgação das informações sobre instrumentos financeiros derivativos (Chen, Dou \& Zou, 2018).

Atualmente no Brasil, os pronunciamentos técnicos que abordam o tratamento contábil dos instrumentos financeiros são os CPC 39 (Instrumentos Financeiros: apresentação), 40 (Instrumentos Financeiros: evidenciação) e 48 (Instrumentos Financeiros). 
Estudos nacionais e internacionais anteriores mostram que o nível de disclosure de instrumentos financeiros tem se mostrado ainda aquém do necessário, o que pode refletir na qualidade da informação contábil (Malaquias \& Zambra, 2017; Machado, Oliveira \& Ribeiro, 2016; Ambrozini, 2014; Adznan \& Nelson, 2014). Com isso, no presente trabalho busca-se preencher uma lacuna no sentido de analisar a relação entre a aderência do conteúdo curricular dos cursos de graduação em ciências contábeis e o disclosure de empresas listadas na B3 sobre instrumentos financeiros derivativos.

Portanto, o objetivo desta pesquisa é analisar a relação entre a aderência do conteúdo curricular dos cursos de graduação em ciências contábeis e o disclosure das empresas listadas na B3, carteira teórica do quarto trimestre de 2017, com base nos pronunciamentos técnicos que tratam sobre instrumentos financeiros derivativos.

Para tanto, elaborou-se um instrumento de coleta de dados com 40 itens baseado nos pronunciamentos técnicos CPC 39 (Instrumentos Financeiros: apresentação), 40 (Instrumentos Financeiros: evidenciação) e 48 (Instrumentos Financeiros). Analisou-se as notas explicativas do ano de 2017 de 44 empresas e projetos pedagógicos/fichas de disciplinas de 250 cursos para o cálculo dos índices de aderência.

Para alcance do objetivo da pesquisa, o artigo é composto por seis partes. Além dessa primeira, a próxima seção apresenta a revisão da literatura nacional e internacional sobre os trabalhos desenvolvidos recentemente nesta mesma linha de pesquisa. A terceira apresenta os procedimentos metodológicos relativos à amostragem utilizada e o tratamento dos dados da pesquisa. A quarta, traz as limitações da pesquisa. Os resultados da pesquisa empírica são apresentados e discutidos na seção 5. Na sexta e última seção são apresentadas as considerações finais, relatados os principais achados desta pesquisa, assim como apresentadas sugestões de trabalhos futuros.

\section{REFERENCIAL TEÓRICO}

Nesta seção são apresentados aspectos teóricos e estudos anteriores sobre disclosure de instrumentos financeiros e ensino desses instrumentos no Brasil.

\subsection{Disclosure de instrumentos financeiros}

No contexto empresarial, pelo simples fato das entidades estarem operando, sujeitam-se a riscos, os quais podem ser administrados com a utilização de instrumentos financeiros derivativos (Malaquias \& Lemes, 2013). Derivativos são instrumentos derivados de um ativo, contratados por pessoas ou instituições nos mercados futuros ou de opções, podendo ser utilizados para realizar hedge (proteção), para gerenciar riscos ou mesmo para especular (Amaral, 2003).

O mercado de derivativos surge naturalmente em um ambiente de instabilidade e crescente volatilidade das taxas de juros e câmbio em todo mundo, a partir do momento em que os que buscam proteção no mercado (os hedgers), transferem o risco para aqueles que procuram assumir tais riscos (os especuladores) (Amaral, 2003).

Esse mercado tem crescido nas últimas três décadas seja no volume de transações seja em termos de disponibilidade de instrumentos financeiros para negociação (Machado, Oliveira \& Ribeiro, 2016). Os mercados de capitais e os modelos de negócios tornaram-se mais complexos e desafiadores, tornando o gerenciamento de riscos uma tarefa mais sofisticada (Kota \& Charumathi, 2018).

O ambiente de negócio complexo e em contínua mudança, trouxe a necessidade de se desenvolver normas de divulgação confiáveis e relevantes para auxiliar os usuários contábeis na tomada de decisão (Kota \& Charumathi, 2018), aumentando a demanda de transparência na divulgação dos instrumentos financeiros derivativos (Chen et al., 2018), como por exemplo, os Pronunciamentos Técnicos CPC 39, 40 e 48 do Comitê de Pronunciamentos Contábeis (CPC). 
Nesse sentido, a regulação contábil sobre a elaboração e divulgação das demonstrações financeiras tem se tornado uma questão global, sendo que a adoção mundial das normas internacionais de contabilidade, conhecidas como International Financial Reporting Standards (IFRS), é um dos maiores eventos regulatórios da história da contabilidade (Leuz \& Wysocki, 2016).

No Brasil, as companhias de capital aberto passaram a ser obrigadas a adotarem as normas internacionais de contabilidade em 2007, com a publicação da Instrução Normativa da CVM no 457/2007 e, por conseguinte, pela promulgação da lei 11.638/2007. A uniformização das regras ficou sob a responsabilidade do CPC, que emitiu, no sentido de normatizar o tratamento contábil dos instrumentos financeiros, os Pronunciamentos Técnicos CPC 14 (com vigência até o ano de 2009), CPC 38 (com vigência até o ano de 2017), CPC 39, CPC 40 e CPC 48.

O disclosure de informações pelas empresas pode ocorrer de duas maneiras, tanto voluntária quanto mandatória, mas de acordo com Mapurunga, Ponte, Coelho e Meneses (2011) é possível que, mesmo havendo a exigibilidade da divulgação de informações acerca dos instrumentos financeiros derivativos, certas empresas podem não divulgá-las. Entretanto, mesmo não as evidenciando no corpo do balanço patrimonial, compete às empresas procederem o seu disclosure nas notas explicativas e, de alguma forma, informando a sua existência aos usuários externos (Malaquias \& Lemes, 2013). Nesse ponto, os órgãos normatizadores assumem importante papel em exigir das empresas um disclosure mínimo (Malaquias \& Lemes, 2013).

Pesquisas anteriores buscaram identificar o nível de disclosure das empresas, bem como verificar os fatores determinantes do nível de disclosure relativos aos instrumentos financeiros. Darós e Borba (2005), dentro da perspectiva de governança corporativa e de acordo com a Instrução Normativa no 235 da Comissão de Valores Mobiliários - CVM, analisaram as Demonstrações Contábeis das vinte maiores empresas brasileiras não financeiras classificadas de acordo com a receita líquida. Como resultado, constataram que a grande maioria das empresas não atendia às determinações da CVM e não evidenciara, de forma clara, concisa e objetiva, as informações referentes às suas operações envolvendo instrumentos financeiros derivativos.

Murcia e Santos (2009) analisaram as 20 maiores empresas não financeiras listadas na Bolsa de Valores de São Paulo para verificarem o impacto da Deliberação CVM no 566/08 e da Instrução CVM no 475/08 na divulgação das suas informações sobre instrumentos financeiros derivativos. Analisaram as demonstrações contábeis das empresas referentes aos exercícios de 2007 e 2008, antes e após a aprovação das referidas normas. Os autores concluíram que houve uma melhora no disclosure das empresas, no entanto ainda não divulgavam integralmente todas as informações exigidas pelas normas.

Mapurunga et al. (2011) verificaram a existência de associação entre a divulgação de informações sobre instrumentos financeiros derivativos e características econômicas de 75 companhias listadas nos Níveis Diferenciados de Governança Corporativa da BM\&FBovespa. Em análise preliminar, observaram que nenhuma das empresas da amostra apresentou todos os itens de evidenciação requeridos, bem como que apenas 11, dos 30 itens de evidenciação, foram divulgados por mais de 50\% das companhias pesquisadas. Em seguida, procederam à Análise de Correspondência (ANACOR) para verificar a associação entre os atributos das firmas e seu nível de evidenciação. Os resultados evidenciaram que os atributos 'Tamanho' e 'Lucro' foram, positivamente, associados à divulgação de informações sobre instrumentos financeiros derivativos.

Malaquias e Lemes (2013) analisaram o nível de disclosure das demonstrações financeiras de empresas brasileiras em relação aos requerimentos dispostos pelo IASB (International Accounting Standards Board) para evidenciação de instrumentos financeiros. Com uma amostra de 24 empresas não financeiras para o período de 2002 a 2006, verificaram que os relatórios fornecidos para o mercado brasileiro possuíam um nível de evidenciação com média estatisticamente inferior ao dos relatórios fornecidos ao mercado norte-americano. Ainda, que empresas de setores regulamenta- 
dos, empresas com maior tempo na emissão de ADRs e empresas maiores apresentaram melhores níveis de evidenciação.

Ambrozini (2014) avaliou o grau de observância das orientações de evidenciação de instrumentos financeiros derivativos emanadas pelo CPC 40 (R1) por parte das companhias abertas brasileiras com ações negociadas na Bolsa de Valores de São Paulo. Por meio de uma pesquisa documental e baseado em um instrumento composto por 23 itens, verificou os relatórios contábeis encerrados em dezembro de 2013 de 45 empresas que operaram com derivativos. Evidências do estudo apontaram que a evidenciação dos instrumentos financeiros derivativos sofreu uma considerável melhora após a introdução das normas IFRS se comparado com estudos realizados antes da adoção destas normas. No entanto, observa que há muito o que se avançar em relação ao conteúdo das notas explicativas relativas aos instrumentos financeiros derivativos exóticos.

Machado, Oliveira e Ribeiro (2016) verificaram como as empresas brasileiras, listadas no IBrX-50 da BM\&FBovespa, evidenciaram os derivativos após a adoção do CPC 14, à luz da Deliberação CVM no. 566/08. Com isso, realizaram uma análise de conteúdo nas notas explicativas, do exercício findo em 2009, de 22 empresas não financeiras, e os resultados indicaram que por um lado as empresas cumpriram de forma mais satisfatória a evidenciação de objetivos e estratégias de gerenciamento de riscos, riscos associados e critérios de avaliação, mas, por outro, os pontos mais críticos abrangeram a evidenciação de resultados obtidos em relação aos objetivos propostos nos riscos associados, registro em contas de ativos e passivos segregados e valores e efeitos no resultado.

Moura, Ziliotto e Mazzioni (2016) analisaram os fatores determinantes da qualidade da informação contábil em companhias abertas listadas na BM\&FBovespa. A qualidade da informação foi avaliada por meio do gerenciamento de resultados e os fatores determinantes observados foram a concentração acionária, governança corporativa, nível de competição do mercado e proporção de ativos intangíveis. Realizaram uma pesquisa descritiva, conduzida por meio de abordagem quantitativa em uma amostra de companhias abertas listadas na BM\&FBovespa, com dados disponíveis no período de 2008 a 2014. Os resultados demostraram que, entre os fatores investigados no período, somente o fator competitividade e a intangibilidade relacionaram significativamente com a qualidade da informação contábil.

No âmbito internacional, Lopes e Rodrigues (2007) verificaram os determinantes das práticas de disclosure relativas à contabilização de instrumentos financeiros pelas companhias listadas na Bolsa de Valores de Lisboa. Baseados nos IAS 32 e IAS 39, emitidos pelo IASB, identificaram que o nível de disclosure está significativamente relacionado com o tamanho das empresas (total de ativos e vendas), tipo do auditor, sua listagem em mercados de capitais externos ao do próprio país e também com o setor da economia.

Drakopoulou (2014) examinou as demonstrações financeiras trimestrais das 30 empresas que compunham o índice Dow Jones, referentes ao ano de 2008, com o objetivo de investigar o nível de atendimento aos requisitos qualitativos e quantitativos do SFAS no 161 - norma que trata da divulgação sobre instrumentos financeiros derivativos e hedge. $\mathrm{O}$ autor concluiu com o estudo que a maioria das empresas falhou em divulgar informações necessárias de hedge de fluxo de caixa, investimentos líquidos em operações estrangeiras e hedges de valor justo.

Adznan e Nelson (2014) investigaram as práticas de divulgação de instrumentos financeiros de empresas listadas da Malásia, mais especificamente o nível de conformidade ao IFRS-07. Como resultados os autores evidenciaram que apesar de as empresas observarem o IFRS-07, houveram vários requisitos omitidos pelas 319 empresas pesquisadas.

Malaquias e Zambra (2017) analisaram o nível de disclosure de instrumentos financeiros de 72 empresas do setor de mineração do Brasil, Chile, Peru e México. Os resultados mostraram que as empresas localizadas no México ofereciam um maior nível de divulgação tanto para o IFRS-07 
quanto para o IFRS-09. Além disso, indicaram que as empesas podem utilizar mais os benefícios da internet para divulgarem mais informações sobre instrumentos financeiros.

Pode-se observar, por todo o exposto, que o nível de disclosure de instrumentos financeiros tem se mostrado ainda aquém do necessário, o que pode refletir na qualidade da informação contábil, e que existem fatores das empresas que o determinam, tais como, tamanho da empresa, lucratividade, intangibilidade, setor da economia, empresas de setores regulamentados, empresas com maior tempo na emissão de ADRs, entre outros.

\subsection{Ensino de instrumentos financeiros no Brasil}

Atualmente, ainda existe uma lacuna no que se refere ao ensino de instrumentos financeiros no Brasil, o que é explorado nesta pesquisa. Conforme Alves, Kronbauer, Ott e Thomaz (2017), a adoção pelo Brasil das normas internacionais de contabilidade não afetou apenas a indústria, comércio e serviços, mas também as Instituições de Ensino Superior, precipuamente aquelas que ofertam o curso de Ciências Contábeis.

Ost e Vendruscolo (2014) analisaram as grades curriculares dos cursos de Ciências Contábeis de IES do Rio Grande do Sul sobre a oferta do conteúdo relativo ao mercado de opções, que é uma espécie de instrumento financeiro derivativo. Dentre as universidades pesquisadas, somente $20 \%$ ofertaram disciplinas ligadas ao mercado de opções.

Alves et al. (2017) realizaram um estudo em que analisaram se os conteúdos de contabilidade constantes nos currículos dos cursos de ciências contábeis em instituições de ensino superior (IES) no Brasil estavam alinhados com os Pronunciamentos Técnicos (CPC), que têm origem nas normas internacionais de contabilidade. A amostra foi composta por 105 instituições que ofereciam o curso de ciências contábeis presencial e disponibilizaram suas ementas e/ou conteúdos programáticos. Concluíram que as IES no Brasil pertencentes à amostra possuíam baixa aderência em relação aos CPCs, e que o ensino da contabilidade harmonizado aos padrões internacionais necessita de melhorias que contemplem um número maior de CPCs nos conteúdos programáticos das IES.

Os autores verificaram que a ocorrência dos CPC's relacionados ao tratamento contábil dos instrumentos financeiros nas ementas e/ou conteúdos programáticos dos cursos das 105 instituições pesquisadas foi baixa. O nível de aderência do CPC 39 foi de 8,6\% (9 instituições) e do 40, 10,5\% (11 instituições). Com isso, pode-se inferir que o ensino de instrumentos financeiros pelos cursos de ciências contábeis pesquisados pode não estar em um patamar adequado de oferta, visto que a grande maioria dos cursos sequer contempla os pronunciamentos contábeis que versam sobre instrumentos financeiros em suas ementas e/ou conteúdos programáticos.

Estudos internacionais demonstraram que alunos de contabilidade percebem que o ensino das normas internacionais de contabilidade pode contribuir para suas carreiras quanto à elaboração das demonstrações contábeis. Helen e Kh (2013) verificaram que os alunos de escolas de negócios de Hong Kong entendem que o conhecimento das normas internacionais de contabilidade poderia auxiliá-los com transações comerciais, reportando o desempenho financeiro e analisando as demonstrações financeiras, sendo que, para isso, os provedores da área de educação promovam a adequação dos seus currículos.

Ainda, de acordo com estudo realizado por Al-Mutairi, Naser e Al-Duwaila (2017) no Kuwait, os alunos das escolas de negócios desse país acreditam que estudar as normas internacionais de contabilidade auxiliam no domínio da mensuração e evidenciação contábil.

Dessa forma, o ensino de instrumentos financeiros pode ter um efeito significativo para a melhora do disclosure, uma vez que a formação de profissionais mais alinhados às normas de contabilidade pode refletir na qualidade das demonstrações contábeis elaboradas por esses profissionais. Assim, pelo exposto, surge a seguinte hipótese: 
Hipótese 1: os itens com baixa frequência nos projetos pedagógicos dos cursos de graduação em ciências contábeis podem também estar entre aqueles de maior fragilidade nas demonstrações financeiras das empresas.

\section{PROCEDIMENTOS METODOLÓGICOS}

Com base nos CPC 39 (Instrumentos Financeiros: apresentação), 40 (Instrumentos Financeiros: evidenciação) e 48 (Instrumentos Financeiros) que abordam o tratamento contábil dos instrumentos financeiros, incluindo derivativos e operações de hedge, elaborou-se um instrumento de coleta de dados com 40 itens. Além disso, os itens foram agrupados em blocos conforme a similaridade do teor de cada item. Estes itens, em tese, deveriam constar nas notas explicativas do ano de 2017 relativas aos exercícios contábeis de 2016 e 2017. Ademais, os projetos pedagógicos e fichas de disciplinas dos cursos de graduação em Ciências Contábeis brasileiros também poderiam conter esses itens, uma vez que poderiam servir como direcionadores para o ensino sobre o tema.

Quanto à verificação do nível de disclosure das empresas, a população do estudo refere-se às empresas listadas na B3, cuja amostra relaciona-se especificamente às empresas do Ibovespa (carteira teórica do quarto trimestre de 2017). Dentre as empresas selecionadas, procedeu-se a exclusão das empresas do setor financeiro, de seguros e de fundos de investimentos, resultando em uma amostra de 44 empresas.

Os cursos foram selecionados por meio de uma busca no portal do MEC (Ministério da Educação), selecionando-se todos os cursos presenciais de graduação em Ciências Contábeis ofertados pelas IES brasileiras que possuíam nota do ENADE do exame de 2015 em outubro de 2017. Com a relação dos cursos, passou-se, então, para a pesquisa dos PPPs e fichas de disciplinas publicados no site da respectiva IES, levantando-se 250 documentos.

Assim, as empresas que mencionaram em suas notas explicativas a utilização de instrumentos financeiros, derivativos e hedge e os cursos que ofertaram este conteúdo receberam a pontuação " 1 " (possui) e aqueles que não mencionaram receberam a pontuação " 0 " (não possui), sendo possível, com este procedimento, identificar a frequência da incidência de empresas que informaram a utilização e de cursos que ofertaram o conteúdo de instrumentos financeiros e calcular o nível de aderência no período observado. A coleta dos dados dos cursos se deu no período de 08 de outubro a 15 de novembro de 2017 e das empresas no período de 18 de outubro a 12 de novembro de 2018.

O nível de aderência é a média das questões que foram indicadas como "1", multiplicada por cem, resultando em um índice de aderência para cada empresa e curso. A equação a seguir ilustra o cálculo:

$$
\text { Índice }=\frac{A}{S} \times 100
$$

\section{Sendo:}

A: total dos itens aderentes

S: soma de todos os itens do instrumento de coleta de dados

No que pertine à análise do disclosure das empresas, como limitações do presente trabalho pode-se elencar a verificação apenas das notas explicativas, uma vez que apesar de as demonstrações contábeis serem o principal meio de divulgação por parte das empresas, outros canais de comunicação também podem ser utilizados. Por esse ângulo, destaca-se que a empresas poderiam utilizar outras formas de divulgação para evidenciar suas operações com instrumentos financeiros, 
derivativos e hedge, dado que na presente pesquisa examinou-se as notas explicativas de 2017 pertencentes às empresas da amostra.

Além disso, o número de empresas analisadas ser restrito à carteira teórica do final do ano de 2017, não sendo possível generalizar os resultados para outras empresas nem para as mesmas quanto a outros períodos.

Já em relação à análise da aderência do conteúdo curricular dos PPPs o presente trabalho limitou-se à análise do currículo formal dos cursos, sendo que há a possibilidade de o conteúdo de instrumentos financeiros ser trabalhado na disciplina de tópicos contemporâneos da Contabilidade, cujo conteúdo programático pode ser definido pelo docente da disciplina e não ser registrado no PPP do curso.

\section{ANÁLISE DOS RESULTADOS}

Passa-se desta forma para a discussão dos resultados. A Tabela 1 apresenta a estatística descritiva dos índices de aderência curricular dos PPPs e das notas explicativas dos cursos e empresas pesquisados.

Tabela 1 - Índice de Aderência dos Cursos

\begin{tabular}{|c|c|c|c|c|c|c|c|c|c|}
\hline \multirow[b]{2}{*}{ Bloco } & \multirow[b]{2}{*}{ Descrição } & \multicolumn{4}{|c|}{ PPP } & \multicolumn{4}{|c|}{ Notas Explicativas } \\
\hline & & Min. & Méd. & Máx. & $\begin{array}{l}\text { Desv. } \\
\text { Pad. }\end{array}$ & Min. & Méd. & Máx. & $\begin{array}{l}\text { Desv. } \\
\text { Pad. }\end{array}$ \\
\hline 1 & $\begin{array}{l}\text { Riscos, Instrumentos financeiros } \\
\text { e suas espécies }\end{array}$ & $0 \%$ & $4,6 \%$ & $42,8 \%$ & $7,8 \%$ & $28,6 \%$ & $43,8 \%$ & $71,4 \%$ & $8,9 \%$ \\
\hline 2 & $\begin{array}{l}\text { Reconhecimento, mensuração e } \\
\text { evidenciação dos instrumentos } \\
\text { financeiros }\end{array}$ & $0 \%$ & $0,4 \%$ & $15,4 \%$ & $1,9 \%$ & $15,4 \%$ & $67,3 \%$ & $84,6 \%$ & $13,3 \%$ \\
\hline 3 & $\begin{array}{l}\text { Instrumentos financeiros deriva- } \\
\text { tivos }\end{array}$ & $0 \%$ & $14,6 \%$ & $71,4 \%$ & $20,6 \%$ & $0 \%$ & $47,4 \%$ & $85,7 \%$ & $28,0 \%$ \\
\hline 4 & $\begin{array}{l}\text { Operações e contabilidade de } \\
\text { hedge }\end{array}$ & $0 \%$ & $1,8 \%$ & $23,1 \%$ & $3,7 \%$ & $0 \%$ & $28,6 \%$ & $69,2 \%$ & $23,9 \%$ \\
\hline 5 & Todos os itens (questões) & $0 \%$ & $4,1 \%$ & $22,5 \%$ & $5,0 \%$ & $10 \%$ & $47,1 \%$ & $70 \%$ & $14,4 \%$ \\
\hline
\end{tabular}

Fonte: Elaborado pelos autores.

Pode-se observar na Tabela 1, o nível de aderência do conteúdo curricular ofertado no geral é baixo $(4,1 \%)$. O bloco 2 (Reconhecimento, mensuração e evidenciação dos instrumentos financeiros) apresentou a menor média do índice de aderência ( $0,4 \%$ ) e o bloco 3 (instrumentos financeiros derivativos) o que apresentou maior índice $(14,6 \%)$. Esses resultados evidenciam que as IES analisadas ainda necessitam abarcar mais itens presentes nos pronunciamentos técnicos sobre de instrumentos financeiros em seus projetos pedagógicos. Esses resultados alinham-se aos achados de Alves et. al. (2017) quanto à oferta de conteúdo relativo a instrumentos financeiros.

Ainda, a Tabela 1 demonstra que a média geral de aderência das empresas pesquisadas foi de $47,1 \%$. O bloco 4 (operações e contabilidade de hedge) apresentou menor nível de aderência (28,6\%) e o maior foi o do bloco $2(67,3 \%)$, ao contrário do que foi apresentado pelos cursos, cujo nível foi o menor. Assim, pouco mais da metade das empresas divulgou em suas notas explicativas informações sobre o reconhecimento, mensuração e evidenciação dos instrumentos financeiros.

Analisando comparativamente a aderência dos cursos e das empresas na Tabela 1, pode-se verificar que não necessariamente os itens com menor aderência de conteúdo dos cursos são os de menor aderência nas notas explicativas, exceto para os itens relacionados ao Bloco 4 (operações e contabilidade de hedge) cujos índices de aderência foram os menores tanto na oferta de seu conteúdo quanto na divulgação de suas informações pelas empresas, rejeitando-se a Hipótese 1. Os itens 
relativos às operações e contabilidade de hedge apresentaram baixo índice de aderência tanto para os PPP quanto para as notas explicativas, sendo em média 1,8\% e 28,6\%, respectivamente.

Ainda, conforme a Tabela 1, as empresas pesquisadas apresentaram, em média, uma aderência de $28,6 \%$ na evidenciação de operações de hedge em suas notas explicativas. Ambrozini (2014) destacou em sua pesquisa um achado negativo em relação à transparência contábil, observando que a evidenciação das operações com hedge foi devidamente apresentada por pouco mais do que a metade das 45 empresas analisadas no estudo.

Resultados condizentes, também, com o estudo de Drakopoulou (2014) no qual concluiu que a maioria das empresas falhou em divulgar informações necessárias de hedge de fluxo de caixa, investimentos líquidos em operações estrangeiras e hedge de valor justo.

Pelo exposto, os resultados apresentados na Tabela 1 evidenciam a necessidade de melhorias na divulgação das informações sobre o tratamento contábil dos instrumentos financeiros derivativos designados como hedge. Ressalta-se que mesmo com a baixa frequência do conteúdo dos instrumentos financeiros nos PPPs, os contadores e profissionais interessados podem fazer cursos e treinamentos para aprimorar seu nível de conhecimento sobre o assunto.

O Quadro 1 relaciona os itens do instrumento que não apresentaram aderência de conteúdo por parte dos cursos pesquisados.

\begin{tabular}{|c|c|}
\hline Bloco & Item (número do item entre parênteses) \\
\hline 1 & $\begin{array}{l}\text { (5) Instrumentos Financeiros Sofisticados; (6) Instrumentos Financeiros Híbridos; (7) Instrumentos Financei- } \\
\text { ros Embutidos; (8) Instrumentos Financeiros Compostos. }\end{array}$ \\
\hline 2 & $\begin{array}{l}\text { (10) Instrumentos Financeiros Disponíveis para Venda; (11) Instrumentos Financeiros Mantidos para Nego- } \\
\text { ciação; (12) Instrumentos Financeiros Mantidos até o Vencimento; (13) Instrumentos Financeiros e Ajustes } \\
\text { de Avaliação Patrimonial; (15) Cálculo do Valor Justo de Instrumentos Financeiros Derivativos; (17) Mensu- } \\
\text { ração de Perda de Crédito de Instrumentos Financeiros; (32) Ativo Financeiro Mensurado ao Valor Justo por } \\
\text { Meio do Resultado; (33) Passivo Financeiro Mensurado ao Valor Justo por Meio do Resultado; (34) Ganho } \\
\text { ou Perda em Ativo ou Passivo Financeiro Mensurado a Valor Justo. }\end{array}$ \\
\hline 4 & $\begin{array}{l}\text { (24) Objeto de Hedge; (26) Contabilização do Hedge de Valor Justo; (27) Contabilização do Hedge de Investi- } \\
\text { mento no Exterior; (28) Hedge de Investimento Líquido; (29) Avaliação da Efetividade do Hedge; (30) Méto- } \\
\text { dos de Avaliação dos Requisitos de Efetividade de Hedge; (31) Mensuração de Inefetividade de Hedge. }\end{array}$ \\
\hline
\end{tabular}

Fonte: Elaborado pelos autores.

Verifica-se no Quadro 01 os itens que são abordados pelos pronunciamentos técnicos sobre instrumentos financeiros e não foram mencionados por nenhuma IES, considerando os 250 cursos analisados. Nesse ponto, apresenta-se uma relação de itens que poderiam ser incluídos pelas IES quando da elaboração e/ou revisão dos seus PPPs. Os itens mais recorrentes no Quadro 01 são referentes às operações de hedge e à classificação de instrumentos financeiros derivativos, tópicos que podem ser considerados relevantes no ensino de instrumentos financeiros no Brasil, pois o mercado de derivativos tem crescido nas últimas décadas (Machado et al., 2016) e as empresas podem se utilizar desses instrumentos para fins de hedge (Amaral, 2003), como forma de administração dos riscos relacionados à atividade empresarial (Malaquias \& Lemes, 2013).

A Tabela 2 evidencia a frequência do número de empresas aderentes aos itens dos pronunciamentos técnicos conforme notas explicativas. 
Tabela 2 - Itens do instrumento com baixa ou nenhuma aderência nas notas explicativas

\begin{tabular}{|c|c|c|c|c|}
\hline Bloco & № Item & Descrição & $\begin{array}{c}\text { № de em- } \\
\text { presas }\end{array}$ & $\%$ \\
\hline \multirow{4}{*}{1} & 5 & Instrumentos Financeiros Sofisticados & 0 & $0 \%$ \\
\hline & 6 & Instrumentos Financeiros Híbridos & 1 & $2 \%$ \\
\hline & 7 & Instrumentos Financeiros Embutidos & 8 & $18 \%$ \\
\hline & 8 & Instrumentos Financeiros Compostos & 1 & $2 \%$ \\
\hline \multirow{3}{*}{2} & 11 & Instrumentos Financeiros Mantidos para Negociação & 9 & $20 \%$ \\
\hline & 13 & Instrumentos Financeiros e Ajustes de Avaliação Patrimonial & 6 & $14 \%$ \\
\hline & 17 & Mensuração de Perda de Crédito de Instrumentos Financeiros & 0 & $0 \%$ \\
\hline \multirow{3}{*}{3} & 37 & Opções de venda (put options) & 8 & $18 \%$ \\
\hline & 39 & Contrato futuro & 7 & $16 \%$ \\
\hline & 40 & Contrato a termo & 10 & $23 \%$ \\
\hline \multirow{9}{*}{4} & 19 & Contabilidade de operações de proteção patrimonial & 0 & $0 \%$ \\
\hline & 23 & Operações de Hedging & 0 & $0 \%$ \\
\hline & 24 & Objeto de Hedge? & 18 & $41 \%$ \\
\hline & 26 & Contabilização do Hedge de Valor Justo & 12 & $27 \%$ \\
\hline & 27 & Contabilização do Hedge de Investimento no Exterior & 3 & $7 \%$ \\
\hline & 28 & Hedge de Investimento Líquido & 8 & $18 \%$ \\
\hline & 29 & Avaliação da Efetividade do Hedge & 19 & $43 \%$ \\
\hline & 30 & Métodos de Avaliação dos Requisitos de Efetividade de Hedge & 4 & $9 \%$ \\
\hline & 31 & Mensuração de Inefetividade de Hedge & 0 & $0 \%$ \\
\hline
\end{tabular}

Fonte: Elaborado pelos autores.

Comparando a Tabela 2 com o Quadro 1, percebe-se que a maioria dos itens (total de 11) que não apresentaram aderência dos PPPs dos cursos aos pronunciamentos técnicos, apresentou baixa ou nenhuma aderência das notas explicativas das empresas da amostra, exceto para os itens 19, 23, 24, 26, 29, 37, 39 e 40 da Tabela 2. Destaca-se que os itens 5, 17 e 31 não apresentaram aderência tanto nos PPPs quanto nas notas explicativas. Dentre os itens com baixa frequência, os que mais foram informados pelas empresas em suas notas explicativas são os itens inerentes à menção do objeto de hedge (18 empresas) e avaliação da efetividade de hedge (19 empresas).

A Tabela 3 demonstra a frequência do número de cursos aderentes aos itens dos pronunciamentos técnicos conforme os PPPs.

Tabela 3 - Frequência dos itens aderentes referente aos PPPs

\begin{tabular}{|c|c|c|c|c|}
\hline Bloco & № Item & Descrição & $\begin{array}{l}\text { № de } \\
\text { cursos }\end{array}$ & $\%$ \\
\hline \multirow{3}{*}{1} & 1 & Gestão de riscos financeiros & 41 & $16 \%$ \\
\hline & 2 & Instrumentos Financeiros & 35 & $14 \%$ \\
\hline & 3 & Classificação de Instrumentos Financeiros & 4 & $2 \%$ \\
\hline \multirow{4}{*}{2} & 4 & Instrumentos Financeiros: Reconhecimento, Mensuração e Evidenciação & 6 & $2 \%$ \\
\hline & 14 & Cálculo do Valor Justo de Instrumentos Financeiros & 2 & $1 \%$ \\
\hline & 16 & Perdas de Crédito de Instrumentos Financeiros & 2 & $1 \%$ \\
\hline & 18 & Provisão para Perdas de Crédito de Instrumentos Financeiros & 2 & $1 \%$ \\
\hline \multirow{5}{*}{3} & 9 & Instrumentos Financeiros Derivativos & 94 & $38 \%$ \\
\hline & 35 & Contrato de Swap & 27 & $11 \%$ \\
\hline & 36 & Contrato de Opções & 46 & $18 \%$ \\
\hline & 37 & Opções de venda (put options) & 5 & $2 \%$ \\
\hline & 38 & Opções de compra (call options) & 5 & $2 \%$ \\
\hline
\end{tabular}




\begin{tabular}{cclcc}
\hline \multicolumn{1}{c}{39} & Contrato futuro & 55 & $22 \%$ \\
& 40 & Contrato a termo & 25 & $10 \%$ \\
\hline \multirow{4}{*}{4} & 19 & Contabilidade de operações de proteção patrimonial & 1 & $0,4 \%$ \\
& 20 & Instrumentos de Hedge & 2 & $1 \%$ \\
& 21 & Contabilidade de Hedge (Hedge Accounting)? & 5 & $2 \%$ \\
22 & Operações de Hedge & 43 & $17 \%$ \\
& 23 & Operações de Hedging & 7 & $3 \%$ \\
& 25 & Contabilização do Hedge de Fluxo de Caixa & 2 & $1 \%$ \\
\hline
\end{tabular}

Fonte: Elaborado pelos autores.

Com base na Tabela 3, os itens mais recorrentes nos PPPs dos cursos pesquisados foram os relativos à menção de gestão de riscos financeiros (41 cursos), instrumentos financeiros (35 cursos), instrumentos financeiros derivativos (94 cursos), operações de hedge (43 cursos), contrato de opções (46 cursos) e contrato futuro (55 cursos).

A Tabela 4 demonstra a frequência do número de empresas aderentes aos itens dos pronunciamentos técnicos conforme as notas explicativas.

Tabela 4 -Frequência dos itens mais aderentes referente às notas explicativas

\begin{tabular}{|c|c|c|c|c|}
\hline Bloco & $\begin{array}{c}\text { № } \\
\text { Itens }\end{array}$ & Descrição & $\begin{array}{c}\text { Frequência } \\
\text { Notas Explicativas } \\
\text { № de empresas }\end{array}$ & $\%$ \\
\hline \multirow{3}{*}{1} & 1 & Gestão de riscos financeiros & 44 & $100 \%$ \\
\hline & 2 & Instrumentos Financeiros & 44 & $100 \%$ \\
\hline & 3 & Classificação de Instrumentos Financeiros & 37 & $84 \%$ \\
\hline \multirow{10}{*}{2} & 4 & $\begin{array}{l}\text { Instrumentos Financeiros: Reconhecimento, Mensuração e Evidencia- } \\
\text { ção }\end{array}$ & 37 & $84 \%$ \\
\hline & 10 & Instrumentos Financeiros Disponíveis para Venda & 29 & $66 \%$ \\
\hline & 12 & Instrumentos Financeiros Mantidos até o Vencimento & 23 & $52 \%$ \\
\hline & 14 & Cálculo do Valor Justo de Instrumentos Financeiros & 43 & $98 \%$ \\
\hline & 15 & Valor Justo de Instrumentos Financeiros Derivativos & 31 & $70 \%$ \\
\hline & 16 & Perdas de Crédito de Instrumentos Financeiros & 44 & $100 \%$ \\
\hline & 18 & Provisão para Perdas de Crédito de Instrumentos Financeiros & 44 & $100 \%$ \\
\hline & 32 & Ativo Financeiro Mensurado ao Valor Justo por Meio do Resultado & 41 & $93 \%$ \\
\hline & 33 & Passivo Financeiro Mensurado ao Valor Justo por Meio do Resultado & 40 & $91 \%$ \\
\hline & 34 & $\begin{array}{l}\text { Ganho ou Perda em Ativo ou Passivo Financeiro Mensurado a Valor } \\
\text { Justo }\end{array}$ & 38 & $86 \%$ \\
\hline \multirow{4}{*}{3} & 9 & Instrumentos Financeiros Derivativos & 30 & $68 \%$ \\
\hline & 35 & Contrato de Swap & 33 & $75 \%$ \\
\hline & 36 & Contrato de Opções & 32 & $73 \%$ \\
\hline & 38 & Opções de compra (call options) & 26 & $59 \%$ \\
\hline \multirow{4}{*}{4} & 20 & Instrumentos de Hedge & 24 & $55 \%$ \\
\hline & 21 & Contabilidade de Hedge (Hedge Accounting) & 24 & $55 \%$ \\
\hline & 22 & Operações de Hedge & 29 & $66 \%$ \\
\hline & 25 & Hedge de Fluxo de Caixa & 23 & $52 \%$ \\
\hline
\end{tabular}

Fonte: Elaborado pelos autores.

Já na Tabela 4, dentre os itens do instrumento que apresentaram maior frequência de empresas, verifica-se que os itens relacionados à divulgação nas notas explicativas de gestão de riscos financeiros, instrumentos financeiros, perdas de créditos de instrumentos financeiros, provisão para perda de créditos de instrumentos financeiros foram evidenciados por todas as empresas da amostra. Em contrapartida, os itens ligados à instrumento de hedge, contabilidade de hedge, hedge de fluxo de caixa e instrumentos financeiros de instrumentos financeiros apresentaram menor frequência 


\section{CONSIDERAÇÕES FINAIS}

O objetivo do presente trabalho foi analisar a relação entre a aderência do conteúdo curricular dos cursos de graduação em ciências contábeis e o disclosure das empresas listadas na B3, carteira teórica do quarto trimestre de 2017, sobre instrumentos financeiros derivativos.

Elaborou-se um instrumento de coleta de dados com 40 itens com base nos pronunciamentos técnicos CPC 39 (Instrumentos Financeiros: apresentação), 40 (Instrumentos Financeiros: evidenciação) e 48 (Instrumentos Financeiros). Analisou-se as notas explicativas do ano de 2017 de 44 empresas e projetos pedagógicos/fichas de disciplinas de 250 cursos para o cálculo dos índices de aderência.

Como principais resultados, verificou-se que não necessariamente os itens com menor aderência de conteúdo dos cursos são os de menor aderência nas notas explicativas, exceto para os itens relacionados a operações e contabilidade de hedge, cujos índices de aderência foram os menores tanto na oferta de seu conteúdo quanto na divulgação de suas informações pelas empresas, rejeitando-se a Hipótese 1.

Os itens relativos às operações e contabilidade de hedge apresentaram baixo índice de aderência tanto para os PPP quanto para as notas explicativas, sendo em média 1,8\% e 28,6\%, respectivamente. Resultados condizentes com os estudos de Ambrozini (2014) e Drakopoulou (2014).

Esses resultados evidenciam a necessidade de melhorias na divulgação das informações sobre o tratamento contábil dos instrumentos financeiros derivativos designados como hedge. Ressalta-se que mesmo com a baixa frequência do conteúdo dos instrumentos financeiros nos PPPs, os contadores e profissionais interessados podem fazer cursos e treinamentos para aprimorar seu nível de conhecimento sobre o assunto.

Para futuras pesquisas sugere-se a verificação realizada neste estudo em períodos posteriores para análise de tendências do comportamento dos índices de aderências apresentados pelas empresas e cursos de graduação em ciências contábeis. Além disso, a realização de pesquisa para a identificação do motivo da baixa evidenciação e oferta de conteúdo sobre operações e contabilidade de hedge pelas empresas e cursos, respectivamente.

\section{REFERÊNCIAS}

Al-Mutairi, A., Naser, K., \& Al-Duwaila, N. (2017). Students' attitudes towards the adoption of international financial reporting standards (IFRS) in Kuwait. Asian Social Science, 13 (5), 85-95. Disponível em: <https://DOI:10.5539/ass.v13n5p85>.

Alves, D. S., Kronbauer, C. A., Ott, E., \& Thomaz, J. L. P. (2017). O ensino dos CPCs nos cursos de ciências contábeis em instituições de ensino superior do Brasil. Revista Contemporânea de Contabilidade, 14 (32), 48-70. Disponível em: <https://periodicos.ufsc.br>.

Amaral, C. A. L. V. do. (2003). Derivativos: o que são e a evolução quanto ao aspecto contábil. Revista Contabilidade E Finanças - USP, São Paulo, 32, 71 - 80. Disponível em: < http://dx.doi.org/10.1590/S1519-70772003000200005>.

Ambrozini, M. A. (2014). Análise do grau de evidenciação das operações com instrumentos financeiros derivativos pelas companhias brasileiras listadas no Ibovespa. Revista Contemporânea de Contabilidade - UFSC, Florianópolis, 11 (24), 25 - 42. Disponível em: $<$ https://doi.org/10.5007/2175-8069.2014v11n24p25>.

Chen, J., Dou, \& Zou, Y. (2018). Information externalities of disclosure regulation: evidence from SFAS 161. SSRN. 
Darós, L. L., \& Borba, J. A. (2005). Evidenciação de instrumentos financeiros derivativos nas demonstrações contábeis: uma análise das empresas brasileiras. Revista de Contabilidade $\mathcal{E}$ Finanças - USP, São Paulo, 39, 68-80. Disponível em: <http://dx.doi.org/10.1590/S1519$70772005000300006>$.

Drakopoulou, V. (2014). Accounting for derivative instruments and hedging activities. Journal of Financial Risk Management, 3, 151-165. Disponível em: <

https://www.researchgate.net/publication/274383702_Accounting_for_Derivative_Instrumen ts_and_Hedging_Activities>.

Helen, W., \& Kh, W. (2013). An empirical study - adoption of international financial reporting standards (IFRS) in Hong Kong education. Journal of Management Research, 5, (4), 98-107. Disponível em: <https://doi.org/10.5296/jmr.v5i4.4256>.

Kota, H. B., \& Charumathi, B. (2018). Determinants of financial derivative disclosures in an emerging economy: a stewardship theory perspective. Australasian Accounting, Business and Finance Journal, 12 (3), 42 - 66. Disponível em: < https://ro.uow.edu.au/aabfj/vol12/iss3/5/>.

Leuz, C., \& Wysocki, P. D. (2016). The economics of disclosure and financial reporting regulation: Evidence and suggestions for future research. Journal of Accounting Research, 54, 525 - 622. Disponível: < https://onlinelibrary.wiley.com/doi/10.1111/1475-679X.12115>.

Lopes, P. T., \& Rodrigues, L. L. (2007) Accounting for financial instruments: an analysis of the determinants of disclosure in the Portuguese stock exchange. The Journal of Accounting, 42, 25 56. Disponível em:

$<$ https://www.researchgate.net/publication/4739828_Accounting_for_financial_instruments_ An_analysis_of_the_determinants_of_disclosure_in_the_Portuguese_stock_exchange $>$.

Machado, D. G., Oliveira, A. F., \& Ribeiro, J. T. (2016). Evidenciação dos derivativos após o CPC 14: um estudo da aderência brasileira em empresas listadas no índice IBRX-50 da BM\&Fbovespa. Sinergia, Rio Grande, 20 (1), 85 - 97. Disponível em: $<$ https://periodicos.furg.br/sinergia/article/view/5454>.

Malaquias, R., \& Lemes, S. (2013). Disclosure of financial instruments according to International Accounting Standards: empirical evidence from Brazilian companies. Brazilian Business Review, 10 (3), 82-107. Disponível em:

$<$ http://www.bbronline.com.br/artigos.asp?sessao=ready\&cod_artigo=82>.

Malaquias, R. F., \& Zambra, P. (2018). Disclosure of financial instruments: practices and challenges of Latin American firms from the mining industry. Research in International Business and Finance, 45, 158-167. Disponível em:

$<$ https://econpapers.repec.org/article/eeeriibaf/v_3a45_3ay_3a2018_3ai_3ac_3ap_3a158167.htm>.

Mapurunga, P. V. R., Ponte, V. M. R., Coelho, A. C. D., \& Meneses, A. F. (2011). Determinantes do nível de disclosure de instrumentos financeiros derivativos em firmas brasileiras. Revista de 
Contabilidade E Finanças - USP, São Paulo, 57 (22), 263 - 278. Disponível em: $<$ http://www.revistas.usp.br/rcf/issue/view/2794>.

Murcia, F. D., \& Santos, A. (2009). Regulação contábil e a divulgação de informações de operações com instrumentos financeiros derivativos: análise do impacto da CVM no 566/08 e da CVM no 475/08 no disclosure das companhias abertas no Brasil. RCO - Revista de Contabilidade e Organizações - FEARP/USP, 3 (6), 3 - 21. Disponível em: $<$ https://www.revistas.usp.br/rco/article/view/34738>.

Moura, G. D., Ziliotto, K., \& Mazzioni, S. (2016). Fatores determinantes da qualidade da informação contábil em companhias abertas listadas na BM\&FBovespa. Revista de Contabilidade e Organizações, 27, 17-30. Disponível em: <http://www.revistas.usp.br/rco/article/view/107810>.

Ost, B. B., \& Vendruscolo, M. I. (2014). Educação de derivativos: práticas e adequação das grades curriculares às possibilidades do mercado de opções em IES gaúchas. Revista de Contabilidade Dom Alberto, 1 (6), 51-67. Disponível em: <http://www.domalberto.edu.br/wpcontent/uploads/2017/08/Educa\%C3\%A7\%C3\%A3o-de-Derivativos-Pr\%C3\%A1ticas-eAdequa \%C3\%A7\%C3\%A3o-das-Grades-Curriculares-\%C3\%A0s-Possibilidades.pdf $>$. 